\title{
Assessing impact of veterinary training on Malaysian farmers
}

\begin{abstract}
This research was initiated to assess the impact of training on farmers' capabilities and its application towards farm practice. This study focused on three Malaysian Department of Veterinary Services training institutions that run programmes for farmers related to livestock farming. A total of 132 farmers and training personnel participated in this study where they were selected as respondents nationwide. A multi-stage approach was used where data were primarily collected using mail survey, telephone survey and personal face-to-face interview. This was then supported by semi-structured interviews with selected individuals using purposive sampling. Analysis of findings suggested that a positive trend emerged indicating the effectiveness of the training programmes with range of variations between those training institutes. However, this paper concludes that an income-focused, uni-linear concept of 'impact' achievement is inadequate to capture training outcomes of these farmers' programmes. Instead, 'impact' can be more appropriately viewed as improvement in the farming techniques as well as being better managers and positive attitude change in their fields. This training impact study concentrated on how those trainings provided to the farmers were able to improve their individual skills, knowledge and attitude.
\end{abstract}

Keyword: Assessment; Veterinary training; Impact of training; Farmers; Malaysia 